\title{
Particularités lexicales du français en Vallée d'Aoste
}

\section{Kamilla Kurbanova}

\section{(2) OpenEdition \\ 12 Journals}

Édition électronique

URL : http://journals.openedition.org/esp/1102

DOI : 10.4000/esp.1102

ISSN : 2532-0319

Éditeur

Centre d'Information sur l'Éducation Bilingue et Plurilingue

Édition imprimée

Date de publication : 1 juin 2017

Pagination : 49-59

ISSN : 1127-266X

\section{Référence électronique}

Kamilla Kurbanova, "Particularités lexicales du français en Vallée d'Aoste », Éducation et sociétés plurilingues [En ligne], 42 | 2017, mis en ligne le 01 février 2018, consulté le 20 avril 2019. URL : http:// journals.openedition.org/esp/1102 ; DOI : 10.4000/esp.1102 


\section{PARTICULARITÉS LEXICALES DU FRANÇAIS EN VALLÉE D'AOSTE (1)}

\section{Kamilla KURBANOVA}

The linguistic questionnaires applied in the Aosta Valley in 2015-2016 allowed us to establish that Aostan French is still dotted with regionalisms that attest to their vitality. The lexemes and collocations confirmed by Aostan speakers of French can be grouped into three main categories: loanwords from Italian and FrancoProvençal, as well as archaisms and lexical neologisms. Thus, the specialized study of the examples contributed by representatives of three generations allowed us not only to elaborate a classification of Aostan regionalisms by their nature, degree of assimilation, etymology, etc., but also to define the trends of the lexical development of Aostan French in the 21st century.

Key words: Aostan French, Aostan regionalism, loanword, archaism, Aostan neologism.

I sondaggi linguistici realizzati in Valle d'Aosta nel 2015-2016 ci hanno permesso di constatare che il francese valdostano è sempre intriso di regionalismi valdostani che fanno prova di una certa vitalità. I lessemi e le espressioni fisse confermati da valdostani francofoni sono divisi in tre categorie principali: italianismi e prestiti dal francoprovenzale, arcaismi e neologismi valdostani. Dunque, l'analisi specifica dei questionari compilati dai rappresentanti di tre generazioni ci ha permesso non solo di elaborare una classificazione dei regionalismi valdostani secondo la loro natura, il livello di assimilazione, l'etimologia, ecc., ma anche di determinare certe tendenze di sviluppo lessicale del francese valdostano nel XXI secolo.

Parole chiave: francese valdostano, regionalismo valdostano, prestito, arcaismo, neologismo valdostano

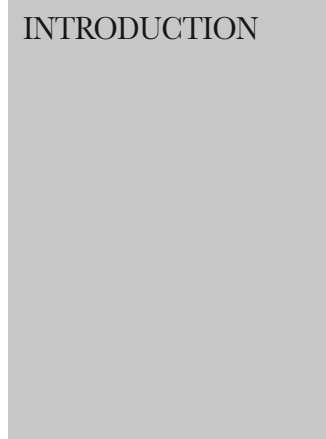
tut spécial d'Italie, se trouve dans le nord-ouest du pays et est contigüe à la France (le département de la Savoie) ainsi qu'à la Suisse (le canton de Valais). Etant la plus petite province italienne avec une population totale de 128000 habitants et une superficie de 3262 km2, la Vallée d'Aoste représente une aire linguistique unique regroupant trois langues romanes à la fois l'italien, le français et le francoprovençal (la langue de type galloromain souvent nommée également patois / patoué valdôtain dont les locuteurs s'appellent, par conséquent, patoisants). Le Statut spécial pour la Vallée d'Aoste (Statuto speciale della Valle d'Aosta), ou Loi constitutionnelle $\mathrm{n}^{\circ} 4$ du 26 février 1948, définit l'autonomie 


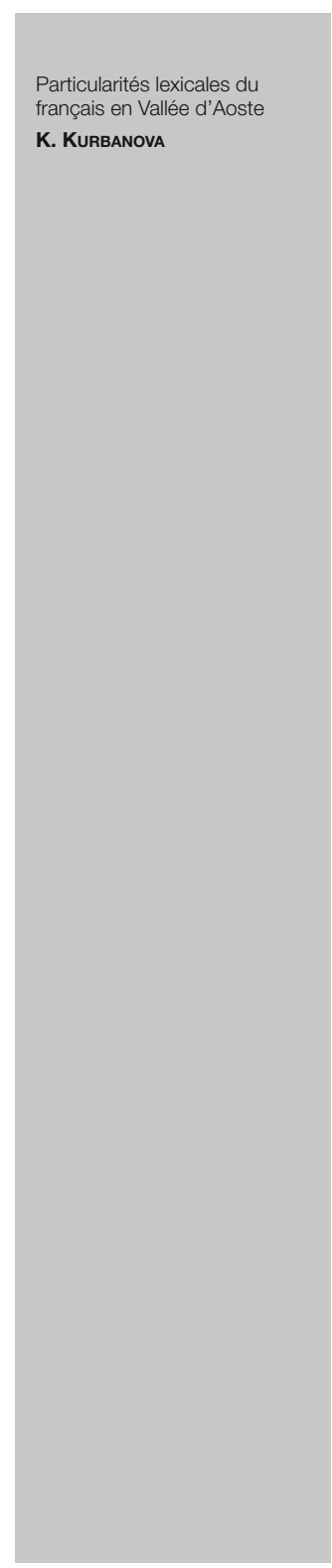

de la région, par contre seulement deux des trois langues nommées ci-dessus ont obtenu le statut officiel - l'italien et le français. La situation plurilingue (bi- ou même trilingue dans certains cas) décrite ne pouvait pas ne pas influencer le français parlé au Val d'Aoste qui est considéré comme une des variétés du français, plus précisément une variété valdôtaine de français répandue sur le territoire de la région. Avant de nous pencher sur la description des «valdôtainismes», il faudrait bien préciser que le fonds lexical de la variété valdôtaine est le français de référence, c'est-à-dire le français standard qui désigne le français dépourvu de tout trait régional. Dans Le dictionnaire de linguistique et des sciences du langage, Dubois définit comme «standard» une forme de langue qui «audelà des variations locales et sociales... s'impose au point d'être employée couramment, comme le meilleur moyen de communication, par des gens susceptibles d'utiliser d'autres formes ou dialectes» (Dubois et al. 2012: 440). La langue standard est «généralement normalisée et soumise à des institutions qui la régentent» (Ibid.), ce qui présuppose qu'elle est décrite dans les dictionnaires, manuels de grammaire, et toutes sortes d'ouvrages de référence. Dans nos recherches, afin de s'assurer que la forme étudiée appartient au français de référence, on consultait surtout des dictionnaires français, comme, par exemple, le Trésor de la Langue Française informatisé ou Le Petit Robert de la langue française.

Mises à part des créations purement valdôtaines, le corpus des particularités du français valdôtain comprend également des mots et des expressions répandus dans d'autres régions de la francophonie. Ces derniers, les régionalismes pas seulement propres au Val d'Aoste, seront notés spécialement.

Il est intéressant d'étudier les particularités lexicales valdôtaines du point de vue de leur étymologie. Quand il s'agit des réalités exclusivement valdôtaines il n'y a pas de concurrence avec le français «standard», vu que ces référents (objets ou êtres) n'existent pas dans la réalité française. La situation devient beaucoup plus curieuse quand un signifié et un signifiant sont bien employés en français de France, mais que les valdôtains préfèrent un autre moyen de dénomination. Quelles sont les sources d'enrichissement de la variété valdôtaine?

Traditionnellement, on distingue trois groupes de mots et d'expressions composant un lexique particulier régional: des emprunts;

des formes anciennes / vieillies;

des néologismes régionaux, ici des néologismes valdôtains formés par dérivation, composition, dérivation impropre, etc. 


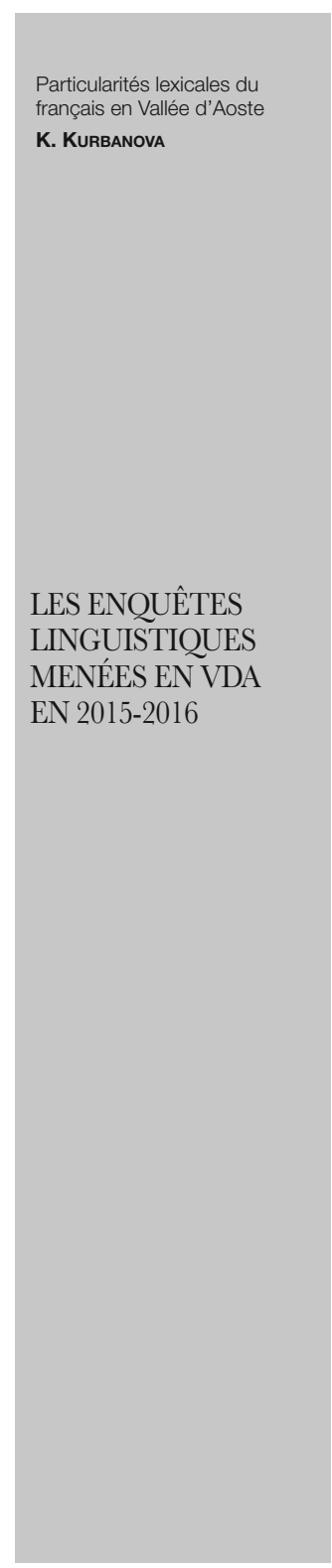

Afin d'éclairer l'état actuel du français parlé au Val d'Aoste, on a entrepris des enquêtes linguistiques au cours des années 20152016 auprès des valdôtains francophones, représentant différents âges et statuts sociaux. Il est à noter qu'une étude précédente des particularités valdôtaines a été organisée par J.-P. Martin dans les années 1980, il y a donc plus de trente ans, il s'agit ainsi d'un travail bien approfondi mais qui date un peu et dont les résultats sont à vérifier et mettre à jour.

En commençant nos recherches, on s'est fixé pour objectif non seulement d'effectuer une description générale du lexique francovaldôtain contemporain, mais aussi de déterminer la dynamique de son évolution pendant les trente dernières années, d'analyser la fréquence d'emploi des valdôtainismes par telle ou telle catégorie de locuteurs, d'établir enfin les tendances principales du développement du français valdôtain au 21 ème siècle.

Le critère obligatoire pour participer aux enquêtes était l'origine valdôtaine des participants (tous nos enquêtés sont nés au Val d'Aoste, majoritairement à Aoste, et y ont habité toute leur vie ou presque - sauf quelques années d'absence ou de départ en mission). Pour pouvoir tirer des conclusions sur l'avenir du français valdôtain, les participants ont été répartis en trois tranches d'âge: groupe 1 - les jeunes jusqu'à 19 ans; groupe 2 - des enquêtés de 20 à 49 ans; groupe 3 - des participants d'âge plus avancé, à partir de 50 ans. En rédigeant nos questionnaires, on a également introduit d'autres facteurs sociolinguistiques, tels que le niveau de formation des enquêtés, leur-s langue-s maternelle-s (2).

Les questionnaires linguistiques contenaient toutes sortes de valdôtainismes soigneusements choisis: chaque classe de valdôtainismes était représentée par un nombre égal d'exemples dans le but de vérifier, premièrement, leur vitalité et d'étudier leur emploi et leurs particularités. Le choix des léxèmes proposé a reposé en grande partie sur la recherche de J.-P. Martin, évoquée ci-dessus, «Description lexicale du français parlé en Vallée d’Aoste» (1984) et sur d'autres articles consacrés à ce sujet, par exemple, celui de J.-M. Kasbarian, «Le français au Val d'Aoste» (2010).

Le questionnaire comportait deux types d'exercices: il fallait choisir une des deux variantes d'une phrase ou expression dont l'une était française, l'autre contenait une particularité valdôtaine; une liste de léxèmes à définir était proposée à la fin, ainsi que des questions de nature sociolinguistique et une colonne spéciale destinée aux commentaires facultatifs (qu'on a reçus en quantité).

Les particularités lexicales du français valdôtain invitent à s'inté- 


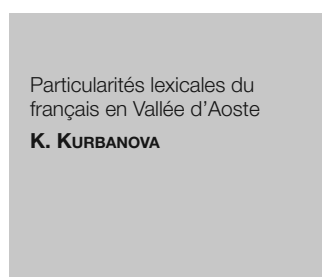

LES EMPRUNTS

resser avant tout aux emprunts car ils représentent une couche essentielle de valdôtainismes, ce qui différencie d'ailleurs cette variété de toutes les autres variétés régionales de français (le français suisse, par exemple, se distingue du français de référence essentiellement par des archaïsmes et non par des emprunts). Malgré son statut officiel, fixé dans le Statut spécial du VDA dont l'Article 38 proclame: «La langue française et la langue italienne sont à parité en Vallée d'Aoste», le français au Val d'Aoste est en réalité beaucoup moins parlé que l'italien et même que le patois, auquel on a recours en troisième lieu après ces deux langues les plus répandues (voir les résultats du sondage linguistique réalisé par la Fondation Emile Chanoux en 2001-2002). Ce sont justement les contacts italien-français et francoprovençal-français qui engendrent la plus grande quantité d'emprunts.

Citons des emprunts à l'italien, souvent appelés italianismes tout court, qui sont entrés dans le français valdôtain et qui subissent différents degrés d'assimilation. Parmi des italianismes non-assimilés, on note pronto (au lieu du français allô), et ape (petit tricycle à moteur), très caractéristique dans la réalité valdôtaine qui n'a pas d'équivalent en français, confirmés par la majorité de nos enquêtés: le pourcentage de réponses positives selon les tranches d'âge était: groupe $1(67 \%), 2(56 \%), 3(75 \%)$. Les italianismes de ce type peuvent être assimilés au français phonétiquement, dans ce cas l'accent est transposé à la dernière syllabe. Il faut se rendre compte que la prononciation varie d'un locuteur à l'autre, il n'y a pas de régularités strictes, cela peut dépendre entre autres du niveau de formation de l'enquêté.

Nous ne donnerons que quelques exemples choisis pour notre article, cependant, il faut comprendre que l'inclusion des mots italiens dans le discours d'un Valdôtain est un processus spontané. Dès qu'un locuteur ne trouve pas de bon équivalent en français il peut recourir à l'italien et cela n'implique pas forcément que ce mot est propre au français valdôtain, mais signifie simplement qu'il est employé occasionnellement.

Le groupe suivant est constitué d'italianismes graphiquement et morphologiquement adaptés dont l'emploi dans le français valdôtain a été confirmé par les résultats de notre questionnaire. Par exemple, le substantif franco-valdôtain tabaquerie est la version assimilée du nom italien tabaccheria et correspond au fran. bureau de tabac. En français de référence existe un verbe arranger (par ex., arranger l'affaire), mais sous l'influence de l'équivalent italien sistemare le verbe français est de plus en plus substitué par systémer (l'affaire) qui est considéré comme un italianisme assimilé. 


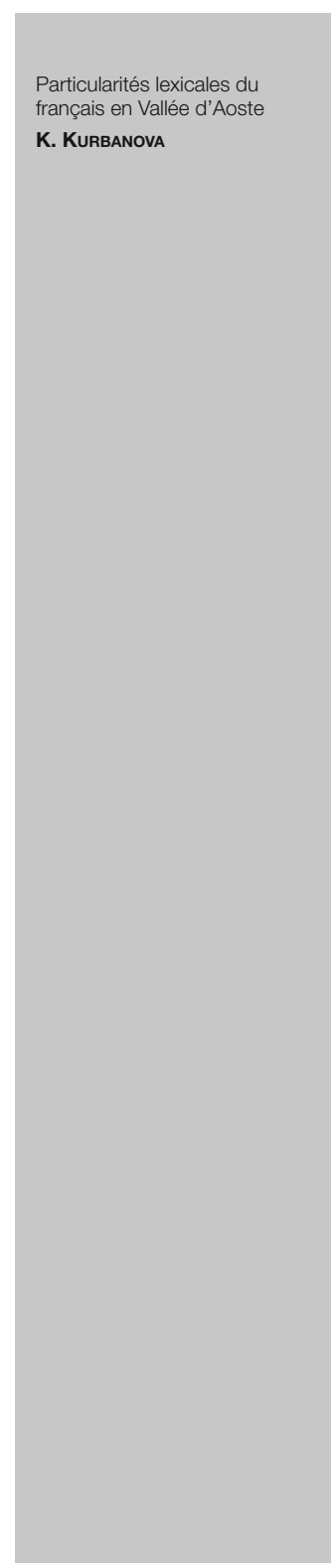

En dehors des emprunts proprement dits, on distingue également des calques. "Quand il s'agit d'un mot simple, le calque se manifeste par l'addition, au sens courant du terme, d'un 'sens' emprunté à la langue B» (Dubois et al. 2012: 74), ainsi, on n'emprunte pas le mot tout entier, mais on rajoute un nouveau sens à un mot déjà existant, cela s'appelle aussi un calque sémantique. Notons que les calques sémantiques sont surtout répandus dans le domaine de l'administration et de la politique valdôtaines. On peut citer par exemple deux substantifs bien français assesseur et junte voulant dire «personne qui siège auprès de qqn, l'assiste dans ses fonctions ou le supplée en son absence; adjoint, assistant», "conseil, assemblée administrative, politique, en Espagne, au Portugal ou en Amérique latine» (les définitions sont tirées du Petit Robert 2011). Par contre, en français valdôtain, ce sont des termes administratifs calqués de l'italien: les mots assessore et Giunta ont défini les sens d'assesseur «responsable d'une subdivision du pouvoir exécutif régional» et funte «organe exécutif (d'une commune, d'une région)» (Ibid.) (s'écrit avec une majuscule dans ce sens-là) et s'emploient maintenant comme équivalents officiels dans les traductions, documents administratifs, etc.

On a aussi traité des calques du type morphosyntaxique, en particulier, le phénomène de calque de combinaisons de mots. L'un des calques de ce genre a eu beaucoup de succès auprès de nos enquêtés. Certains «puristes» qui évitaient soigneusement toutes sortes d'emprunts proposés dans les corpus du questionnaire ont quand même confirmé l'usage de ce calque: au fran. bougie de voiture les valdôtains préfèrent une expression chandelle de voiture, c'està-dire ils remplacent un substantif français très répandu bougie par un autre mot français beaucoup plus rare chandelle, en calquant l'expression italienne candela della macchina. Le parallélisme avec l'expression italienne analogue suscite, à ce qu'il paraît, un taux très élevé d'emploi, les résultats selon les tranches d'âge étant: groupe $1(83 \%) 2$ (78\%), 3 (63\%). D'après les données affichées, on peut constater que la jeune génération est plus portée à l'usage des emprunts.

Pour terminer avec les calques, il faudrait noter encore ceux de type phraséologique dont chaque élément est traduit littéralement: toucher le ciel du doigt est un calque phraséologique de l'italien toccare il cielo col dito «être débordant de joie », C'est mon clou! - de l'ital. È il mio chiodo fisso! «C'est mon obsession!».

Ayant décrit les principaux types d'emprunts et de calques de l'italien connus dans la variété valdôtaine de français, on pourrait dégager une tendance pour la fréquence plus notable des italia- 


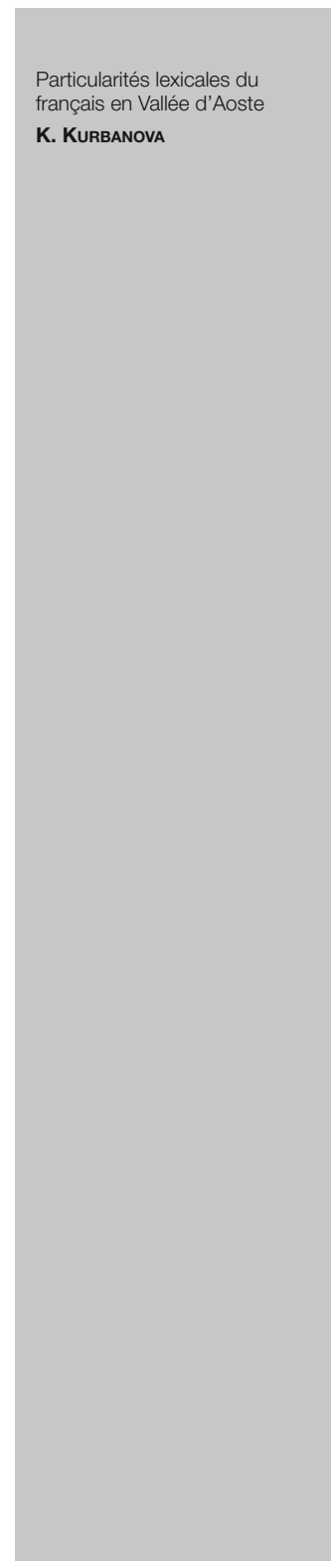

nismes chez les jeunes qui ont préféré une variante avec un emprunt à l'italien dans $40 \%$ des cas, les représentants du groupe 2 dans $38 \%$ des cas, et ceux du groupe 3 dans $35 \%$ des cas.

La deuxième source d'emprunt reste toujours le francoprovençal, ou le patois. Il faut rappeler que la question sur la langue maternelle faisait partie de la fiche signalétique de notre questionnaire et $39 \%$ des enquêtés, autrement dit, plus de tiers de participants, ont noté le patois comme leur première langue ou bien le patois et l'italien.

Parmi les emprunts directs du patois valdôtain, on doit absolument citer l'exemple de rabeilleur qui vient du francoprov. rhabilleur I rabeilleur, rabeilleusa et qui s'est tellement bien installé dans l'usage des francophones valdôtains qu'ils ne doutent même pas que ce mot n'est pas français à l'origine. En français de référence, c'est le subst. rebouteux qui est employé. En parlant de la répartition des réponses, remarquons que la grande majorité des enquêtés a préféré la variante valdôtaine, ainsi les représentants du groupe 1 ont choisi rabeilleur et pas rebouteux dans $67 \%$ des cas, ceux du groupe 2 à $89 \%$, et ceux du groupe 3 à $82 \%$.

Comme on l'a déjà noté, à la fin de notre questionnaire, on a proposé une liste de mots auxquels il fallait donner une définition et tous ont plus ou moins bien accompli cette tâche. Entre autres, le mot mayen («pâturage à mi-montagne») a largement confirmé sa vitalité, n'étant pas un lexème uniquement valdôtain: il est bien représenté dans les patois valaisans d'où il s'est transféré dans le français de la Vallée d'Aoste, mais il est aussi connu en Savoie et en Suisse (Thibault, Knecht 2012: 511). Le mot est d'ailleurs traduit en français par le même mot (maven), ce qui démontre que pour les lexicographes valdôtains il s'agit d'un mot indubitablement français (Chenal et al. 2007).

Le francoprovençal a également engendré des calques phraséologiques comme laver la chemise à quelqu'un («critiquer quelqu'un») du patois lavé la tsemise a quatsun ou avoir la tête montée dans la lune («être ivre») du patois avei la têta vià pe la leuna.

En ce qui concerne les emprunts au francoprovençal, il faut mettre en relief que contrairement à toute attente, malgré l'urbanisation générale, la formation plus approfondie et plus répandue, etc, on n'a pas observé de baisse brutale dans l'usage des emprunts au patois: selon la totalité de réponses on peut constater que les plus jeunes ont reconnu et ont pu définir 35\% des mots et expressions proposés, le groupe moyen $41 \%$, le groupe plus âgé $39 \%$. Le taux moyen de reconnaissance et d'emploi peut être expliqué par plusieurs raisons. Il y avait notamment des enquêtés 


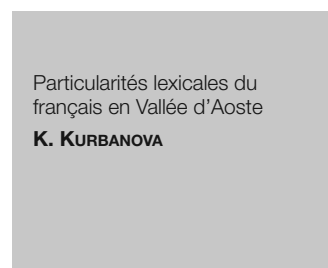

LES ARCHAÏSMES

qui nous ont écrit dans leurs commentaires qu'ils comprenaient très bien les emprunts choisis, mais dans le cadre du questionnaire, ils optaient plutôt pour une variante en français «standard». Comme dans la vie, ils cherchent à éviter des formes empruntées au francoprovençal quand ils parlent avec un francophone qu'ils ne connaissent pas parce qu'ils ne peuvent pas être sûrs que celuici soit patoisant.

Le français valdôtain est aussi marqué par l'emploi de formes archaiques ou vieillies même si ces dernières ne constituent pas le trait distinctif de la variété. On appelle archaïsme un mot ou une construction syntaxique française qui s'emploie toujours en français de la Vallée d'Aoste contrairement au français de référence où ces formes / tournures sont perçues comme archaïques de nos jours et n'appartiennent plus à l'usage courant. Il faut bien souligner la relativité de cette notion, parce que ces formes ne paraissent vieillies que dans une synchronie, appartenant à un état de langue ancien, mais auparavant celles-là étaient bien employées dans le langage courant français. Quant aux valdôtains, les formes concernées sont tout à fait habituelles pour eux, ainsi que pour tous les autres locuteurs des variétés de français.

Dans le cadre de cet article, on ne traite pas tous les types d'archaïsmes (de prononciation, grammaticaux, syntaxiques, etc), on se concentre plutôt sur les archaïsmes lexicaux.

Premièrement, on parlera des archaïsmes qui sont connus dans presque toute la francophonie et dont les plus répandus sont: - les anciens noms de nombre employés au Val d'Aoste et également dans d'autres pays: septante «soixante-dix» (Belgique, Suisse, est de la France, R. D. du Congo, Rwanda), huitante «quatrevingt» (Suisse), nonante "quatre-vingt-dix» (Belgique, Suisse, Rwanda, Burundi, R. D. du Congo). Il est à noter qu'historiquement le système vigésimal était plus ancien par rapport au système décimal, par contre, au cours du 17ème siècle, suite aux travaux de C.F. de Vaugelas et à la normalisation entreprise par l'Académie française, le compte par vingt s'est définitivement fixé en France, le compte par dix étant déconseillé à l'époque. Les formes septante, huitante, nonante sont à présent perçues comme vieillies ou régionales par la majorité des français.

- les anciens noms des repas: déjeuner «petit déjeuner», dîner/ dinée «déjeuner», souper «dîner», répandus dans les pays francophones nommés ci-dessus ainsi qu'au Québec.

Dans le répertoire d'archaïsmes valdôtains «purs», citons des exemples qui sont vieillis non seulement de forme mais aussi de 


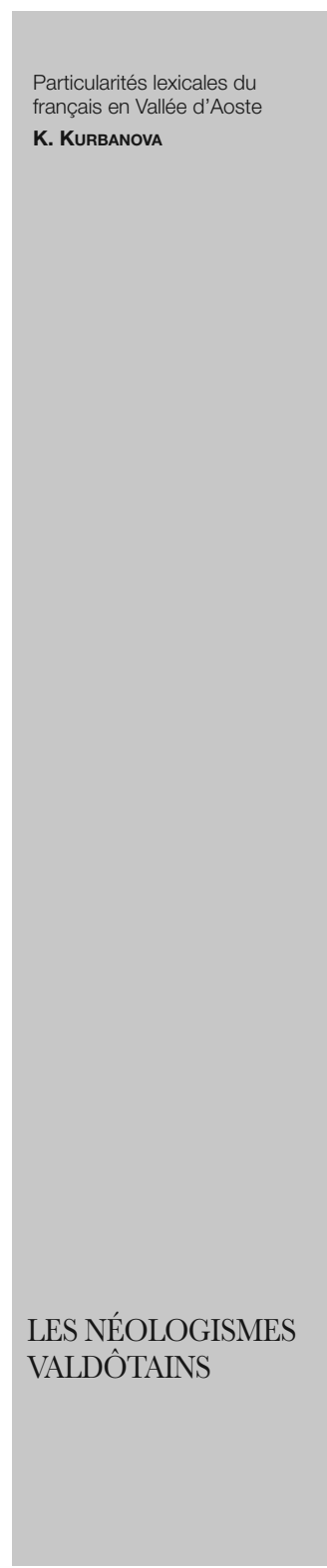

notion: entre autres, notre questionnaire comportait un substantif poëlle "chambre chauffée» du fran. vx poêle qui a attiré l'attention de certains enquêtés, ceux-là nous ont donné des commentaires supplémentaires expliquant qu'ils comprenaient bien le mot, mais qu'actuellement cette réalité - la partie de maison indiquée n'existait plus.

En tant qu'archaïsme lexico-syntaxique, on pourrait décrire le régime vieilli du verbe quitter, plus précisément, quitter qch / qqn à qqn dans le sens «laisser qch / qqn à qqn» qui était d'usage courant en français de référence jusqu'au 15ème siècle. Maintenant son emploi a été confirmé par certains francophones valdôtains: entre les deux phrases 1) «elle a quitté ses enfants à sa mère» et 2) «elle a laissé ses enfants à sa mère», $44 \%$ des participants du groupe 3 ont choisi la forme archaïque, alors que les représentants du groupe 1 et du groupe 2 ont accepté cette forme seulement dans $17 \%$ et $33 \%$ des cas.

Selon les résultats de notre enquête, on peut dire que l'emploi des archaïsmes en français valdôtain est en baisse, le taux de reconnaissance de ces formes est assez modeste: seulement $7 \%$ chez les représentants du groupe $1,18 \%$ du groupe $2,20 \%$ du groupe 3 . Leur emploi dépend de l'âge des locuteurs; ceux qui ont plus 50 ans les ont acceptés plus que les autres mais cette supériorité n'est pas considérable. Ainsi on peut conclure que les traits archaïques ne sont pas typiques du français parlé en Vallée d'Aoste autant que d'autres variétés.

Cependant, cela n'implique pas que les valdôtains cèdent en quantité et en diversité des archaïsmes aux autres francophones: selon les témoignages des jeunes valdôtains, les formes archaïques (tels qu'on a les déjà évoquées, les anciens noms de nombre, par ex.) ont un caractère symbolique pour eux. Connaissant très bien les analogues français contemporains, qu'ils apprennent depuis l'école maternelle jusqu'à l'université, certains représentants de la jeunesse valdôtaine font un choix conscient en employant septante, huitante, nonante, ce qui peut être considéré dans le contexte actuel comme une affirmation de l'identité culturelle.

La troisième classe de valdôtainismes qui constitue l'originalité de cette variété est illustrée par des néologismes, ou créations valdôtaines, c'est-à-dire de nouveaux mots formés selon les modèles productifs français ou de nouvelles tournures valdôtaines qui ne sont repérées ni en France, ni dans d'autres régions de la francophonie.

La création des néologismes valdôtains se base avant tout sur dif- 


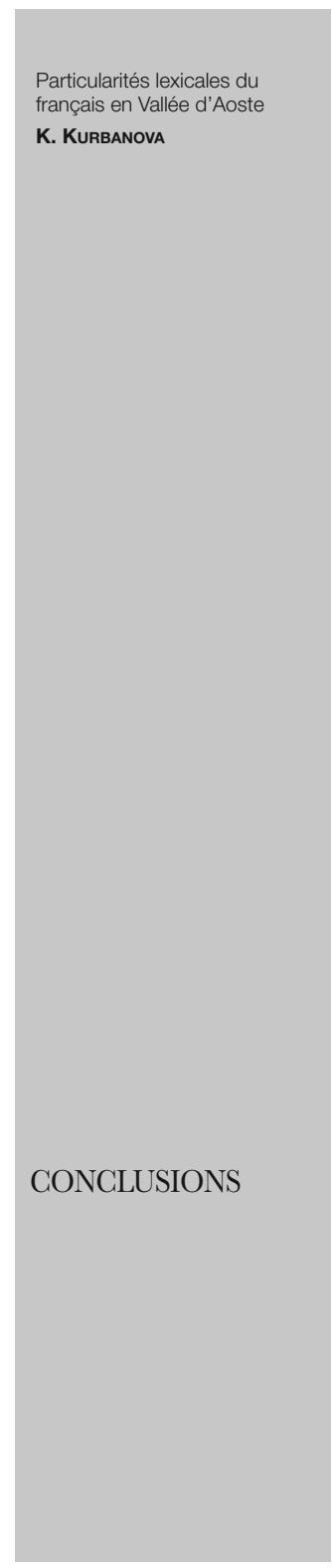

férents types de dérivation. La préfixation est à l'origine, par exemple, de la formation du substantif prémaman qui veut dire «enceinte». La création du néologisme valdôtain loyage - «loyer»peut avoir au moins deux interprétations: il peut être vu comme un dérivé formé à l'aide du suffixe -age, donc par suffixation, sinon on peut y saisir l'influence du patois valdôtain dans lequel le substatif «loyer» se dit loyadzo (Armand 2013: 137), ce qui a probablement provoqué son alignement en français valdôtain.

La composition est à la base de la formation de mère-fille «mère célibataire»: dans cet exemple, on met l'accent sur l'inversion des composants par rapport à la source française - un mot français fille-mère (marqué comme vieilli ou péj. dans les dictionnaires) lui servant effectivement de modèle; en français valdôtain, par contre, il n'y a pas de connotation négative dans ce sens-là.

La créativité de la variété a été attestée au niveau des expressions valdôtaines qui ne sont connues que dans cette région. L'expression figée suivante s'est révélée la plus répandue: comprendre à grosses tranches, ce qui signifie comprendre globalement». Dans notre questionnaire, la tournure était donnée dans la phrase Le français, il le comprenait à grosses tranches qui a été confirmée par la moitié des participants du groupe 1, par 67\% du groupe 2, par $44 \%$ du groupe 3 .

Malgré le fait que les néologismes valdôtains d'après leur forme sont très similaires aux mots français d'origine, ils sont cependant vite reconnus par les locuteurs natifs français et n'appartiennent donc pas à l'usage «standard». Les données statistiques concernant les créations valdôtaines et leur emploi sont de 18\% de reconnaissance chez les représentants du groupe 1, 34\% chez ceux du groupe 2, 29\% du groupe 3, ce qui nous fait penser que ce type de valdôtainismes n'est pas très productif actuellement et la création de nouveaux mots et expressions est en baisse.

Certains points méritent d'être soulignés. Tout d'abord, cette recherche a permis de distinguer les particularités lexicales de la variété valdôtaine en les confirmant auprès des enquêtés valdôtains et en les classifiant en trois catégories: emprunts à l'italien et au francoprovençal, archaïsmes et néologismes. Les exemples choisis de chaque groupe de valdôtainismes ont prouvé leur vitalité, ce qui est déjà très important.

En ce qui concerne la dynamique de l'évolution du français valdôtain pendant les trente dernières années, il faut dire qu'en comparant les résultats de notre recherche et la liste de termes de J.-P. Martin, on a découvert qu'à peu près $30 \%$ des unités lexicales 


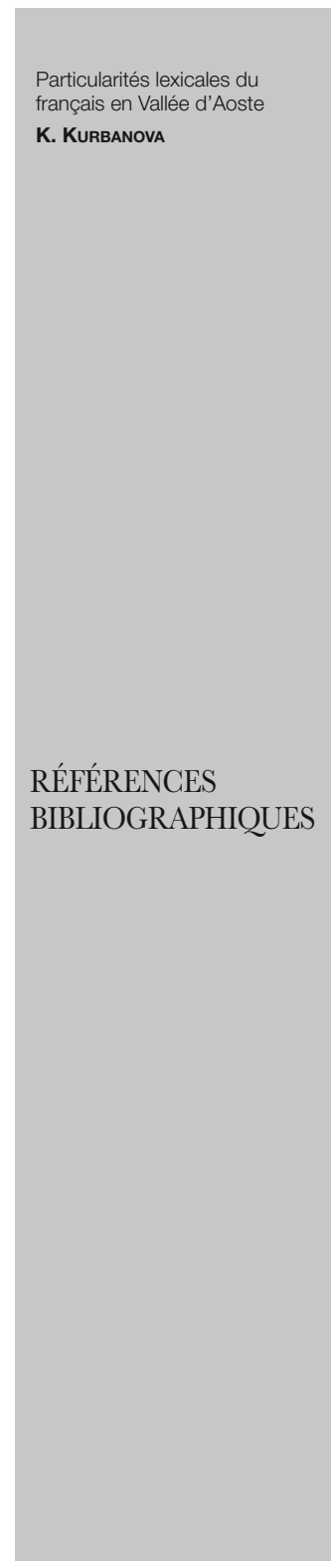

marquées par J.-P. Martin comme valdôtaines ne sont plus attestées lors de la nouvelle enquête.

Selon les données générales obtenues, on peut conclure que le plus grand taux de fréquence a été démontré par les emprunts à l'italien et au francoprovençal. L'indice total des emprunts au patois est de 39\% (sans différence de tranches d'âge), celui des italianismes - 38\% - est un peu moins élevé. En deuxième lieu se sont retrouvés les néologismes valdôtains (28\%), les archaïsmes donnant le résultat le plus modeste $(20 \%)$.

Autrement dit, deux tendances contradictoires se dégagent: d'un côté, les archaïsmes et les créations valdôtaines sont en baisse, par contre, on assiste à un processus de hausse constante des emprunts.

La conclusion générale est que le français qui coexiste comme troisième langue pour la majorité de la population après l'italien et le francoprovençal, conserve ses particularités régionales jusqu'à présent. Le tiers du corpus lexical de la variété comporte des régionalismes, indépendamment de l'âge des locuteurs, de leur niveau de formation et même de leur langue-s maternelle-s.

ARMAND F. 2013. Petit dictionnaire du francoprovençal valdôtain. Piccolo dizionario del francoprovenzale valdostano. Petsou dichonnero de patoué valdôten. Aosta: Le Château Edizioni.

BERARD E. (eds.). 2005. Dictionnaire du patois valdôtain. Aoste: Le Château Edizioni.

CHENAL A., VAUTHERIN R. 2007. Nouveau Dictionnaire de Patois Valdôtain, Aoste: Musumeci.

DUBOIS J., GIACOMO M., GUESPIN L., MARCELLESI G., MARCELLESI J.-B., MEVEL J.-P. (eds.). 2012. Le dictionnaire de linguistique et des sciences du langage. Paris: Larousse.

KASBARIAN J.-M. 2010. Le français au Val d'Aoste, pp. 337 351 in D. DE ROBILLARD, M. BENIAMINO (eds.), Le français dans l'espace francophone, Vol. 1. Paris: Éditions Champion.

Le Petit Robert de la langue française. Dictionnaires Le Robert, 2011.

MARTIN J.-P. 1984. Description lexicale du français parlé en Vallée d'Aoste. Aoste: Musumeci Editeur.

MARTIN J.-P. 1979. Le français parlé en Vallée d'Aoste et sa situation linguistique par rapport à l'italien, pp. 271-284 in A. 


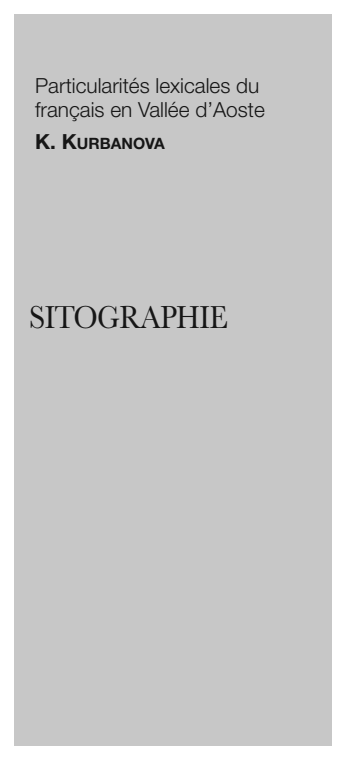

VALDMAN, R. CHAUDENSON, G. MANESSY (eds.), Le français hors de France. Paris: H. Champion.

PÖLL B. 2002. Francophonie périphériques. Histoire, statut et profil des principales variétés du français hors de France. Paris: L'Harmattan.

THIBAULT A., KNECHT P. (eds.) 2012. Dictionnaire suisse romand. Particularités lexicales du français contemporain. Nouvelle édition revue et augmentée. Genève: Éditions Zoé.

Fondation Emile Chanoux. Résultats du sondage linguistique: http://www.fondchanoux.org/sondagelinguistiqueq.aspx

Trésor de la Langue Française informatisé:

http://atilf.atilf.fr/tlf.htm

Statut spécial pour la Vallée d'Aoste: http://www.consiglio.regione.vda.it/fr/app/statuto

\section{NOTES}

(1) L'auteur de l'article tient à exprimer ses remerciements pour leur aide précieuse dans la réalisation de l'enquête, à l'Assessorat de l'Education et de la Culture de la Région autonome Vallée d'Aoste, au Bureau régional pour l'Ethnologie et la Linguistique, au Comité des Traditions Valdôtaines, à la Fondation Emile Chanoux, au Centre d'Etudes Francoprovençales, aux Archives historiques régionales, au Fonds valdôtain de la Bibliothèque régionale d'Aoste, à la Bibliothèque de l'Université de la Vallée d'Aoste, ainsi qu'aux participants au questionnaire et à tous ceux qui ont prêté attention à ce travail.

(2) Une trentaine de personnes ont été enquêtées au total. La majorité est née à Aoste et y a vécu toute sa vie ou presque, ce qui nous a permis de constater que tous avaient fait leurs études primaires et secondaires dans la région. Quant à la formation supérieure, elle concerne un nombre relativement important de participants: $56 \%$ du groupe 2 (20-49 ans ); $37 \%$ du groupe 3 (50 ans ou +). La question de la langue maternelle a particulièrement attiré notre attention, parce que dans certains cas nos enquêtés ne pouvaient pas en citer une seule. En gros, les deux tiers d'enquêtés ont déclaré l'italien en tant que langue maternelle, notamment la moitié des participants les plus jeunes, $77 \%$ du groupe intermédiaire et $56 \%$ du troisième groupe $(50+$ ). Le reste des informateurs ont indiqué le patois (ou le patois et l'italien) comme première.s langue.s, sans parler des autres langues maîtrisées (étrangères ou régionales). Bien évidemment tous les participants étaient francophones, ce qui était indispensable pour pouvoir participer à l'enquête. L'emploi et surtout la fréquence des valdôtainismes lexicaux varient selon ces groupes sociolinguistiques; ce sujet mérite par conséquent une analyse et une description à part. 